\title{
Referenciação aos cuidados hospitalares através de um processo informatizado
}

Hugo Rocha, ${ }^{1}$ Raquel Braga, ${ }^{2}$ Carla Silva, ${ }^{1}$ Sílvia Martins, ${ }^{1}$ Sofia Faria ${ }^{1}$

\section{RESUMO}

Objetivos: Em 2003, um estudo realizado em Matosinhos, previamente à informatização do sistema, determinou uma taxa de referenciação hospitalar de 10,1\%, com referenciações maioritariamente de qualidade média e um tempo médio para a marcação de consulta de um a seis meses. Através de um processo de referenciação informatizado, este estudo pretende determinar a taxa de referenciação hospitalar de todos os médicos de família do ACeS de Matosinhos, em 2016, avaliar a qualidade das referenciações e analisar os tempos de resposta hospitalar.

Métodos: Estudo transversal, analítico, efetuado na Unidade Local de Saúde de Matosinhos, sendo a unidade de investigação as cartas de referenciação dos utentes referenciados ao hospital de referência, em 2016. Amostra aleatória simples, representativa da população $(n=649)$. Análise com recurso a estatística descritiva e analítica (Kruskall-Wallis).

Resultados: A taxa de referenciação foi de 7,7\%. A idade mediana da amostra foi de 53 anos; $53,7 \%$ do sexo feminino. As especialidades mais referenciadas foram oftalmologia (18,0\%), ortopedia (14,4\%) e cirurgia geral (9,9\%). Da amostra de referenciações, $54,1 \%$ das cartas tiveram finalidade diagnóstica; $66,3 \%$ foram de razoável e 31,1\% de boa qualidade; $6,6 \%$ foram recusadas, sendo o principal motivo «Sem critérios clínicos»; e 90,6\% foram triadas em 30 dias. A qualidade das cartas de referenciação influenciou o tempo de efetivação da consulta $(p=0,003)$.

Conclusões: Treze anos após o primeiro estudo verificam-se mudanças: maior transparência, melhoria na identificação e legibilidade da informação das referenciações. Verificou-se uma melhoria da qualidade das referenciações. Houve alterações ao nível das especialidades mais referenciadas, sugerindo que, havendo melhor oferta de cuidados, surge uma maior procura. A informatização das referenciações permitiu maior descriminação dos tempos de espera para efetivação da consulta. Foi possível seguir todo o trajeto de marcação das consultas hospitalares, com monitorização dos tempos de triagem, agendamento e efetivação, embora com limitações inerentes a erros nos processos de agendamento e utilização do ALERT-CTH ${ }^{\circledR}$. Trata-se de um estudo robusto, com validade interna, sendo importante verificar em que medida é que os resultados podem ser semelhantes noutros contextos.

Palavras-chave: Referenciação; Cuidados de saúde primários; Cuidados de saúde secundários; Hospitais.

\section{INTRODUÇÃO}

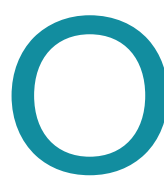
médico de família (MF) constitui o primeiro ponto de contacto do utente com o sistema de saúde, assumindo um papel primordial na avaliação clínica e resolução da maioria dos motivos de consulta. Tem também a responsabilidade de gerir eficientemente os recursos de saúde, coordenando a prestação de cuidados, trabalhando com outros profissionais no contexto dos cui-

1. Médico Interno de Medicina Geral e Familiar. USF Lagoa, ULS Matosinhos. 2. Médica Assistente Graduada em Medicina Geral e Familiar. USF Lagoa, ULS Matosinhos. dados de saúde primários (CSP) e fazendo a interface com os cuidados hospitalares, quando necessário. ${ }^{1} \mathrm{~A}$ boa articulação entre estes dois níveis de cuidados afigura-se fundamental, no sentido de assegurar uma melhor qualidade dos cuidados prestados, garantindo a continuidade dos mesmos e minorando os custos económicos associados. Garante uma melhor gestão das listas de espera, reduzindo a duplicação ou sobreposição de cuidados, bem como a partilha de responsabilidade clínica entre os vários intervenientes.

De uma forma geral, excetuando situações informais ou casos de urgência em que a solicitação de consulta 
pode ser efetuada de forma verbal direta ou por meio de contacto telefónico, o pedido de uma consulta, entendida como a solicitação de uma consulta ao nível dos cuidados hospitalares, com determinado propósito clínico, que implica o envio de informação relevante e completa, será doravante tratado como referenciação e deve ser feito por escrito (nota, $e$-mail ou carta). A referenciação deve conter, no mínimo, os principais problemas do doente, os principais achados e estudos efetuados, medicações prescritas e o motivo de referenciação. ${ }^{2}$

As razões para referenciar podem ser o estabelecimento do diagnóstico, o tratamento, o pedido de opinião acerca de algum achado do exame clínico ou de resultados de exames complementares de diagnóstico ou para tranquilização do paciente. ${ }^{2} \mathrm{O}$ Royal College of General Practitioners considera que a emissão de informação clínica completa, que possibilite aos médicos dos cuidados hospitalares uma avaliação eficiente dos problemas do doente, é um critério de uma prática clínica de qualidade, bem como o encorajamento de uma política de transferência e tratamento da informação que possibilite a continuidade de cuidados. ${ }^{3} \mathrm{~A}$ não explicitação do motivo de referenciação pode gerar perda de tempo em torno de investigações desnecessárias e sobretudo respostas desadequadas por parte dos cuidados hospitalares. O motivo de referenciação deve ser clarificado com o doente para que ele adeque as expectativas relativamente à consulta pretendida ou mesmo, em certos casos, para que não sinta a referenciação como uma rejeição por parte do $\mathrm{MF}^{2}$

A referenciação constitui um dos processos chave na articulação eficiente entre níveis de cuidados. Deve ocorrer sempre que o MF perceciona uma necessidade de cuidados de saúde que estão para além da capacidade de gestão em CSP, envolvendo a partilha de responsabilidade clínica com os profissionais de saúde hospitalares. ${ }^{4}$ Uma referenciação incorreta pode afetar a qualidade dos cuidados, levando à prestação de cuidados fragmentados e inapropriados, custo-inefetivos, aumentando o risco e também a angústia do utente. ${ }^{5}$

A informação veiculada na referenciação e o tempo de espera entre o pedido efetuado pelo MF e a marcação da consulta ao nível hospitalar têm sido alguns dos fatores apontados como tendo maior influência na qualidade da comunicação entre os CSP e os cuidados hospitalares.
Alguns estudos têm sido realizados neste âmbito, embora ainda sejam escassos, sendo descritas taxas de referenciação variáveis entre $5,56 \%$ e $5,87 \%,{ }^{6-7}$ sendo as especialidades cirúrgicas as mais referenciadas, ${ }^{7-8} \mathrm{com}$ tempos médios até à consulta variáveis entre quatro e doze semanas. ${ }^{6-8}$

Um estudo publicado em 2006, realizado no Centro de Saúde (CS) da Senhora da Hora (Matosinhos), ${ }^{9}$ determinou uma taxa de referenciação desta unidade aos cuidados hospitalares, no ano de 2003, de 10,1\%, tendo demonstrado que as especialidades mais referenciadas foram otorrinolaringologia, ginecologia e cirurgia geral. A maioria (65,3\%) das cartas de referenciação avaliadas foi considerada de qualidade razoável e o tempo médio de espera entre o pedido e a efetivação da consulta foi, na maioria dos casos, de um a seis meses, com uma informação de retorno só presente em $26,3 \%$ dos casos. Este estudo foi realizado previamente à informatização generalizada dos processos de referenciação.

Atualmente, o acesso à informação clínica contida em diversos sistemas de informação, acessíveis através do Registo de Saúde Eletrónico (RSE) e o sistema de referenciação ALERT-CTH®, poderão contribuir para a melhoria da articulação entre os diferentes níveis de cuidados, facilitando uma comunicação em rede e possibilitando acesso a informação clínica do paciente mais detalhada.

Decorridos treze anos após o estudo realizado em Matosinhos (Senhora da Hora) ${ }^{9}$ muitas mudanças ocorreram, quer a nível tecnológico quer a nível dos recursos humanos, das práticas clínicas e dos sistemas de informação. Importa reavaliar alguns aspetos da articulação de cuidados, comparando com os resultados encontrados no estudo prévio, relativos ao ano de 2003, mas ampliando a população do estudo a todas as referenciações com origem no Agrupamento de Centros de Saúde (ACeS) de Matosinhos para o principal hospital de referência, o Hospital Pedro Hispano (HPH).

O objetivo deste estudo é determinar a taxa de referenciação do ACeS de Matosinhos, no ano de 2016; avaliar as características e a qualidade da informação enviada nos pedidos de referenciação; e analisar os tempos de resposta hospitalar às referenciações efetuadas: tempo de espera na triagem de consultas, resposta às solicitações, tempo de marcação de consulta e tempo 
de efetivação da consulta. É também objetivo do estudo determinar a existência de associação entre a idade dos médicos de família e a qualidade das cartas enviadas, bem como a existência de associação entre a qualidade das cartas e o tempo de efetivação de consultas hospitalares.

\section{MÉTODOS}

Foi efetuado um estudo observacional, transversal, com componente analítica, tendo a recolha de dados decorrido entre abril e agosto de 2017. A unidade de investigação é constituída pelas cartas de referenciação enviadas por todas as Unidades de Saúde do ACeS de Matosinhos [dez Unidades de Saúde Familiar (USF) e quatro Unidades de Cuidados de Saúde Personalizados (UCSP)], que cobrem uma população de cerca de 180.000 utentes, ao HPH, durante o ano de 2016.

Para o cálculo da taxa de referenciação foi considerado o número total de referenciações recebidas no HPH provenientes das unidades de saúde do ACeS de Matosinhos a dividir pelo número total de consultas efetuadas pelo mesmo ACeS, nesse mesmo ano (incluindo domicílios). Foram excluídas as referenciações a outras instituições hospitalares, ao serviço de urgência, consultas e atos não médicos e pedidos de tratamento de medicina física e reabilitação a centros convencionados, que não o HPH.

Para caracterizar as cartas de referenciação enviadas e avaliar a sua qualidade, bem como para determinar os tempos de resposta, nomeadamente o tempo de execução de triagem, o tempo de agendamento da consulta e o tempo até à efetivação da consulta, minorando o impacto de erros informáticos, de classificação na resposta do triador ou dos serviços administrativos do hospital, detetados no ficheiro original retirado do programa ALERT-CTH®, procedeu-se a uma amostragem das cartas de referenciação.

Após determinação do tamanho amostral através do programa Epi Info ${ }^{\circledR}$ (assumindo um nível de confiança de $95 \%$, uma margem de erro de $5 \%$ e uma prevalência esperada para um evento desconhecido de $50 \%$ ) foi efetuada uma amostragem por seleção aleatória simples das cartas de referenciação, através do programa informático randomizelist ${ }^{\circledR}$, tendo como base a listagem de todos os pedidos de referenciação via ALERT-CTH® realizados durante o ano de 2016 pelos MF do
ACeS de Matosinhos. A análise das cartas de referenciação ( $n=649$, correspondendo a $2 \%$ das 30.423 cartas de referenciação aos HPH no ano de 2016) foi efetuada pelos autores, tendo-se definido previamente a uniformização dos critérios de recolha de dados e sua codificação num suporte informático, Microsoft ${ }^{\circledR}$ Excel2007, sendo os dados tratados com recurso ao Software Statistical Package for the Social Sciences (SPSS), versão $21.0 ®$. A qualidade de $5 \%$ das cartas de referenciação foi avaliada, inicialmente, por todos os autores, tendo posteriormente sido avaliada individualmente. Em casos de dúvida foram realizadas reuniões de consenso entre todos os autores.

Foi utilizada estatística descritiva e inferencial, tendo sido analisada a associação entre a idade dos médicos e a qualidade das cartas enviadas, bem como a existência de associação entre a qualidade das cartas e o tempo de efetivação da consulta hospitalar, recorrendo ao teste de Kruskall-Wallis.

As variáveis analisadas foram as seguintes: sexo e idade dos utentes; número de utentes referenciados pelo MF durante o ano de 2016; idade do médico referenciador; especialidade referenciada; motivo de referenciação (operacionalizado em «diagnóstico», «tratamento", «reavaliação», «segunda opinião», «motivo não apurado», «outros»); qualidade dos pedidos de referenciação; resposta ao pedido de referenciação (operacionalizada em «recusa» ou «agendamento de consulta»); motivo de recusa do pedido ( «sem critérios clínicos»; «informação insuficiente»; «erro na utilização do ALERT-CTH ${ }^{\circledR}$ »; «proposta de terapêutica/exame complementar de diagnóstico»; «referenciação para outra especialidade»; «referenciação duplicada»; «outros»; «não aplicável»); tempo de execução de triagem (número de dias completos entre a data de emissão do pedido de referenciação [D0] e a data de resposta ao mesmo pela triagem hospitalar [D1]); tempo de agendamento da consulta (número de dias completos entre D0 e a data de agendamento da consulta hospital [D2]); tempo até à efetivação da consulta (número de dias completos entre a D0 e a data de efetivação da consulta hospitalar [D3]).

Para avaliação da qualidade de referenciação caracterizou-se cada pedido relativamente à presença de antecedentes familiares, antecedentes pessoais, medicação habitual, história da doença atual, exame objeti- 
vo/exames complementares de diagnóstico, motivo de referenciação/hipóteses diagnósticas. Posteriormente classificou-se cada uma das referenciações em três níveis de qualidade: boa qualidade, se verificada a presença de cinco ou mais critérios, com presença obrigatória da doença atual e motivo de referenciação/hipóteses diagnósticas; qualidade razoável, se dois a quatro critérios presentes ou dois a cinco critérios, sem doença atual descrita ou sem motivo de referenciação/hipóteses diagnósticas explícitos; e má qualidade, se apenas presente um critério dos enunciados anteriormente.

O trabalho foi submetido à Comissão de Ética da Unidade Local de Saúde de Matosinhos e aos Conselhos Clínico e de Administração, tendo obtido parecer favorável à sua execução.

\section{RESULTADOS}

Realizaram-se 35.169 referenciações aos cuidados de saúde secundários, de 1 de janeiro a 31 de dezembro de 2016, sendo que 30.423 destas foram direcionadas ao HPH. No mesmo ano foram realizadas 396.153 consultas pelo ACeS, incluindo domicílios, o que corresponde a uma taxa de referenciação total aos cuidados hospitalares de 8,9\% e uma taxa de referenciação ao HPH de 7,7\% para o ano de 2016.

Do total de referenciações realizadas ao HPH selecionou-se uma amostra aleatória de 649 referenciações ( $2 \%$ do total de referenciações), que foram analisadas na sua totalidade.

No Quadro I encontram-se representados dados relativos à população e amostra das referenciações do ano de 2016, no que diz respeito a variáveis demográficas dos utentes referenciados (idade e sexo) e número de referenciações para cada especialidade, podendo-se observar que a amostra é muito semelhante à população no que concerne a todas as variáveis em estudo.

Verificamos que oftalmologia $(18,0 \%)$, ortopedia $(14,4 \%)$ e cirurgia geral $(9,9 \%)$ foram as especialidades mais solicitadas. As três especialidades menos referenciadas ao HPH foram imuno-hemoterapia $(0,5 \%)$, medicina física e de reabilitação $(0,4 \%)$ e infeciologia $(0,3 \%)$.

\section{Características das referenciações}

Da análise individual de cada referenciação verificou-se que o motivo mais frequente de referenciação é a orientação diagnóstica em 54,1\% dos casos, seguido da finalidade terapêutica (32,7\%) (Figura 1).

A mediana de idades dos médicos referenciadores foi de 46 anos (AIQ=22), tendo o médico mais jovem 28 anos e o mais velho 66 anos (Figura 2).

Relativamente ao conteúdo das referenciações constatou-se que a descrição da doença atual que motivou a referenciação estava presente na maioria dos casos, contrariamente aos dados relativos aos antecedentes familiares e à medicação habitual (Quadro II).

Tendo em conta o conteúdo das referenciações e os critérios definidos previamente, a qualidade das referenciações foi considerada como «Razoável» em 66,3\%, «Boa» em 31,1\% e «Má» em 2,6\% das cartas.

Quando avaliada a associação entre a idade dos médicos de família e a qualidade das cartas enviadas verificou-se que quanto mais jovem o médico, melhor a qualidade da referenciação $\left(\chi^{2}=16,921 ; p=0,000 ; \mathrm{df}=2\right)$ (Figura 3).

Quanto melhor a qualidade da carta de referenciação mais curto foi o tempo até à efetivação da consulta, com significado estatístico $\left(\chi^{2}=11,520 ; p=0,003 ; \mathrm{df}=2\right)$ (Figura 4).

\section{Características das respostas dos cuidados hospitalares}

Em resposta às referenciações efetuadas $(n=649)$ foram realizados 606 agendamentos de consulta $(93,4 \%)$ e o pedido de consulta foi recusado em 43 casos $(6,6 \%$ das referenciações).

Tendo por base o número de pedidos de consulta para cada especialidade, as três especialidades que recusaram maior número de pedidos de consulta foram medicina física e reabilitação, cardiologia e hematologia clínica (Quadro II).

As especialidades de cirurgia geral/ambulatório, cirurgia maxilo-facial, infeciologia, endocrinologia, imunoalergologia, nefrologia, pediatria, psiquiatria e psiquiatria da infância e da adolescência não recusaram qualquer pedido de consulta, procedendo ao agendamento da totalidade dos pedidos.

Relativamente aos motivos de recusa, o mais comum foi «Sem critérios clínicos» (Figura 5). Os motivos contemplados em «Outros» dizem respeito a: recusa de consulta de obstetrícia (termo), pois o parto já tinha ocorrido; recusa de consulta de cirurgia geral, sem des- 


\begin{tabular}{|c|c|c|c|c|c|}
\hline \multicolumn{3}{|l|}{ População } & \multicolumn{3}{|l|}{ Amostra } \\
\hline Especialidade & $n$ & $\%$ & & $n$ & $\%$ \\
\hline Oftalmologia & 5.474 & $18,0 \%$ & Oftalmologia & 127 & $19,6 \%$ \\
\hline Ortopedia & 4.380 & $14,4 \%$ & Ortopedia & 93 & $14,3 \%$ \\
\hline Cirurgia geral & 3.018 & $9,9 \%$ & Cirurgia geral & 68 & $10,5 \%$ \\
\hline Otorrinolaringologia & 2.541 & $8,4 \%$ & Otorrinolaringologia & 58 & $8,9 \%$ \\
\hline Ginecologia & 2.070 & $6,8 \%$ & Ginecologia & 41 & $6,3 \%$ \\
\hline Dermatologia/rastreio teledermatológico & 1.867 & $6,1 \%$ & Psiquiatria/consulta geral & 31 & $4,8 \%$ \\
\hline Psiquiatria/consulta geral & 1.353 & $4,5 \%$ & Dermatologia/rastreio teledermatológico & 30 & $4,6 \%$ \\
\hline Urologia & 1.089 & $3,6 \%$ & Urologia & 25 & $3,9 \%$ \\
\hline Obstetrícia & 933 & $3,1 \%$ & Pediatria & 21 & $3,2 \%$ \\
\hline Cirurgia plástica e reconstrutiva & 850 & $2,8 \%$ & Obstetrícia & 19 & $2,9 \%$ \\
\hline Medicina interna & 812 & $2,7 \%$ & Medicina interna & 17 & $2,6 \%$ \\
\hline Pediatria & 795 & $2,6 \%$ & Cirurgia plástica e reconstrutiva & 16 & $2,5 \%$ \\
\hline Pneumologia & 754 & $2,5 \%$ & Cardiologia & 15 & $2,3 \%$ \\
\hline Neurologia & 691 & $2,3 \%$ & Pneumologia & 14 & $2,2 \%$ \\
\hline Cardiologia & 653 & $2,2 \%$ & Imunoalergologia & 12 & $1,8 \%$ \\
\hline Endocrinologia & 566 & $1,9 \%$ & Neurologia & 11 & $1,7 \%$ \\
\hline Imunoalergologia & 432 & $1,4 \%$ & Endocrinologia & 9 & $1,4 \%$ \\
\hline Psiquiatria da infância e adolescência & 428 & $1,4 \%$ & Anestesiologia & 7 & $1,1 \%$ \\
\hline Gastrenterologia & 404 & $1,3 \%$ & Gastrenterologia & 6 & $0,9 \%$ \\
\hline Anestesiologia & 219 & $0,7 \%$ & Imuno-hemoterapia & 5 & $0,8 \%$ \\
\hline Cirurgia geral/ambulatório & 219 & $0,7 \%$ & Nefrologia & 5 & $0,8 \%$ \\
\hline Cirurgia maxilo-facial & 200 & $0,7 \%$ & Hematologia clínica & 4 & $0,6 \%$ \\
\hline Hematologia & 179 & $0,6 \%$ & Psiquiatria da infância e da adolescência & 4 & $0,6 \%$ \\
\hline Nefrologia & 158 & $0,5 \%$ & Cirurgia geral/ambulatório & 4 & $0,6 \%$ \\
\hline Imuno-hemoterapia & 138 & $0,5 \%$ & Cirurgia maxilo-facial & 3 & $0,5 \%$ \\
\hline Medicina física e reabilitação & 111 & $0,4 \%$ & Infeciologia & 2 & $0,3 \%$ \\
\hline Infeciologia & 89 & $0,3 \%$ & Medicina física e de reabilitação/fisiatria & 2 & $0,3 \%$ \\
\hline \multicolumn{6}{|l|}{ Sexo } \\
\hline Masculino & 14.086 & $46,3 \%$ & & 288 & $44,4 \%$ \\
\hline Feminino & 16.337 & $53,7 \%$ & & 361 & $55,6 \%$ \\
\hline TOTAL & 30.423 & $100,0 \%$ & & 649 & $100,0 \%$ \\
\hline \multicolumn{6}{|l|}{ Idade (anos) } \\
\hline Mínima & 0 & & & 0 & \\
\hline Máxima & 100 & & & 93 & \\
\hline Mediana (AIQ) & & $52(34)$ & & & $53(32)$ \\
\hline
\end{tabular}

Legenda: $\mathrm{AIQ}=$ amplitude interquartil.

Fonte: Dados do ALERT-CTH ${ }^{\circledR}$ 


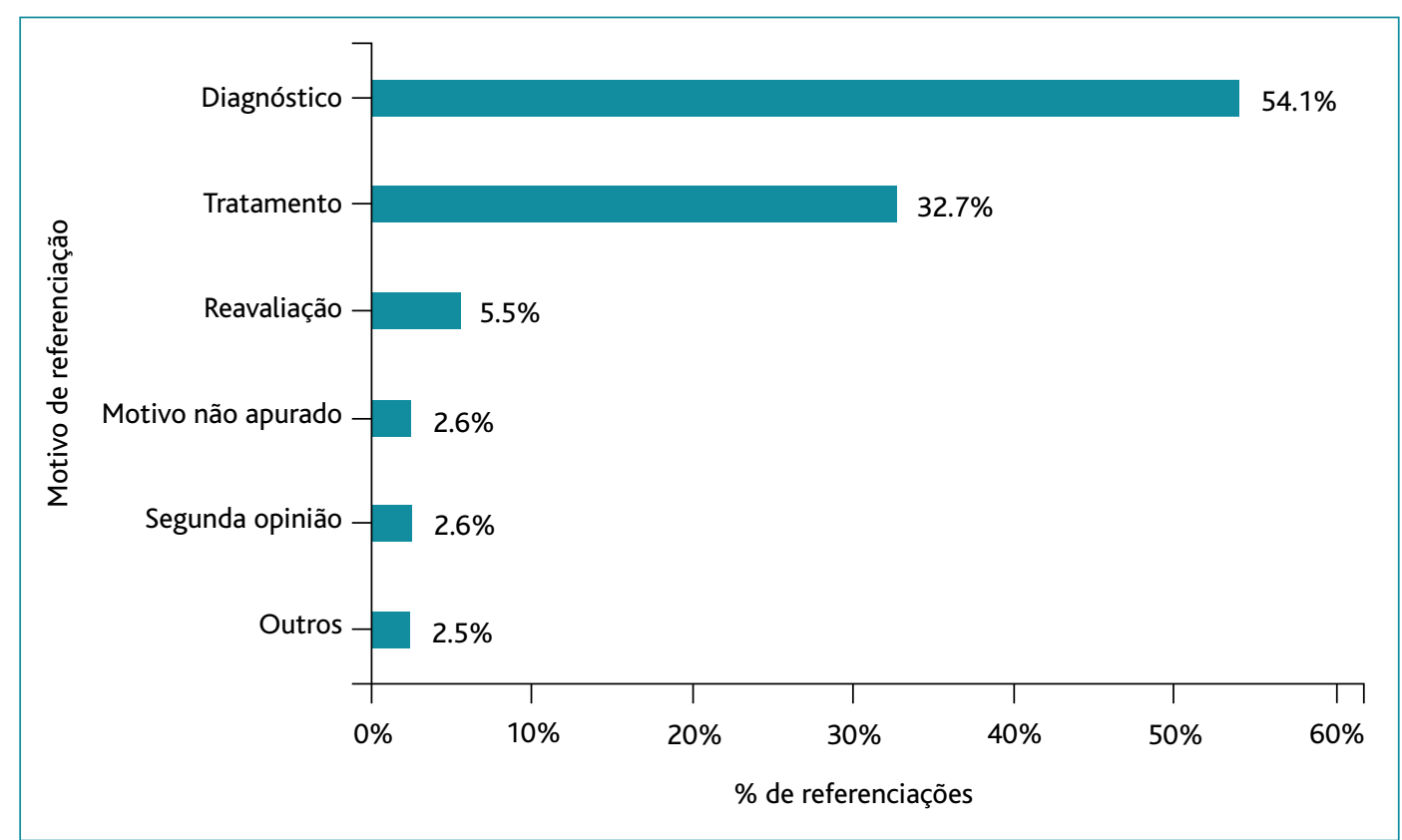

Figura 1. Motivos de referenciação expressos nas cartas enviadas pelos médicos de família do ACeS de Matosinhos ao Hospital Pedro Hispano.

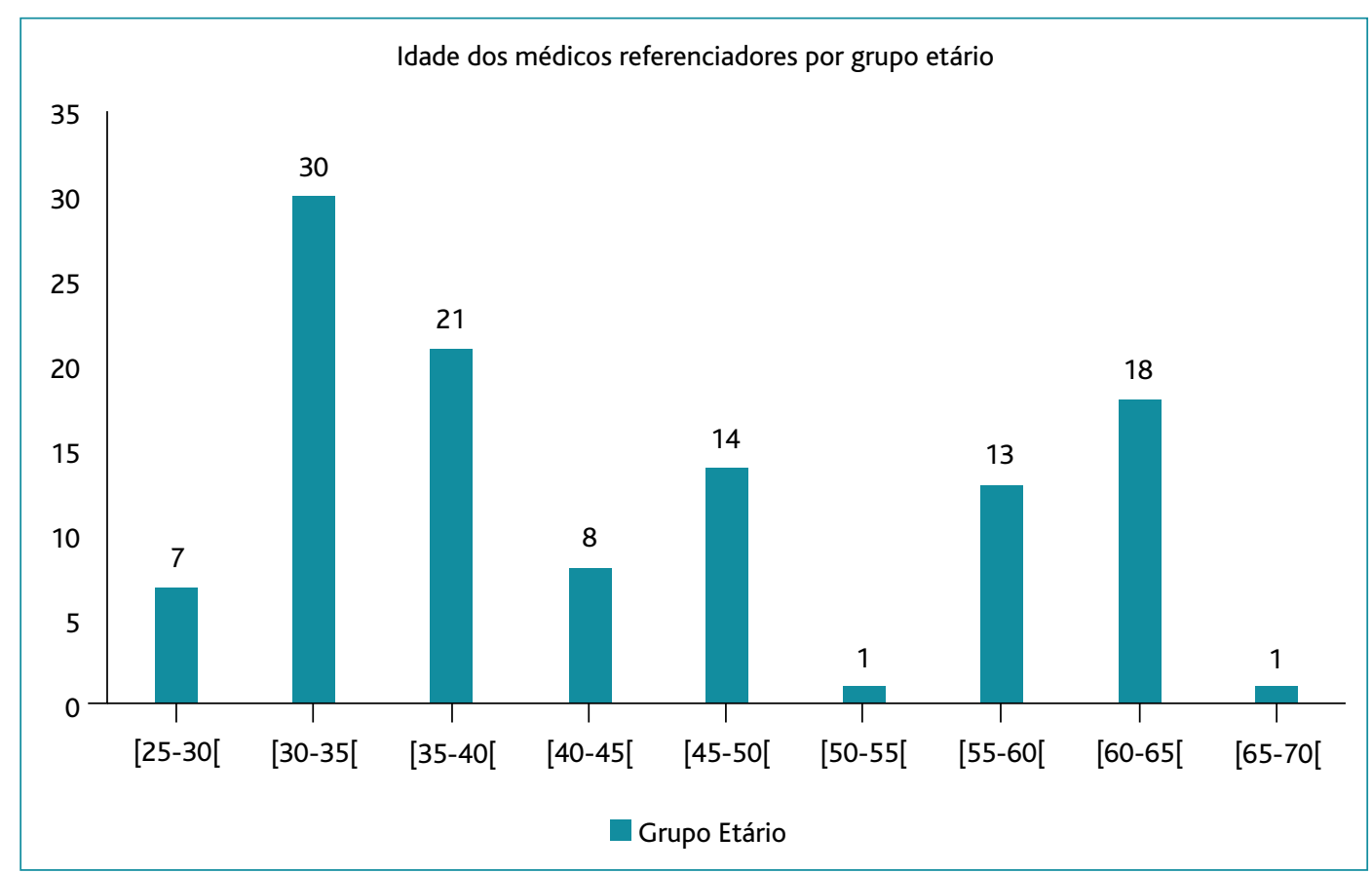

Figura 2. Idade dos médicos de família do ACeS de Matosinhos, por grupo etário [mediana=46 anos (AIQ=22 anos)].

crição do motivo de recusa; recusa de consulta de oftalmologia, pois a referenciação tinha sido feita com o objetivo de execução de cirurgia refrativa, não realizada no hospital de referência. 


\begin{tabular}{|c|c|c|}
\hline \multicolumn{3}{|c|}{$\begin{array}{l}\text { QUADRO II. Conteúdos da informação presente nas } \\
\text { cartas de referenciação }\end{array}$} \\
\hline Conteúdos presentes nas referenciações & $N$ & $\%$ \\
\hline Doença atual & 627 & 96,6 \\
\hline Motivo de referenciação & 598 & 92,1 \\
\hline EO/ECD & 480 & 74,0 \\
\hline Antecedentes pessoais & 461 & 71,0 \\
\hline Medicação habitual & 254 & 39,1 \\
\hline Antecedentes familiares & 59 & 9,1 \\
\hline
\end{tabular}

Legenda: $\mathrm{N}$ = número absoluto; $\mathrm{EO}=$ exame objetivo; $\mathrm{ECD}=$ exames complementares de diagnóstico.

periores a 90 dias nas especialidades de dermatologia/rastreio teledermatológico (319 dias), otorrinolaringologia (186 dias), urologia (92 dias) e nefrologia (92 dias).

Verifica-se que as especialidades mais céleres a triar os pedidos de referenciação são medicina física e de reabilitação (mediana: 1 dia) e psiquiatria da infância e da adolescência (mediana: 1; AIQ=3; IC95\% [0-12]). Por outro lado, verifica-se que as especialidades de otorrinolaringologia (mediana: 45; AIQ=89; IC95\% [10-55]) e infeciologia (mediana: 21; AIQ=59; IC95\% [16-26] foram as que mais tardaram na execução da triagem.

\section{Tempo de agendamento e tempo de efetivação da} consulta hospitalar

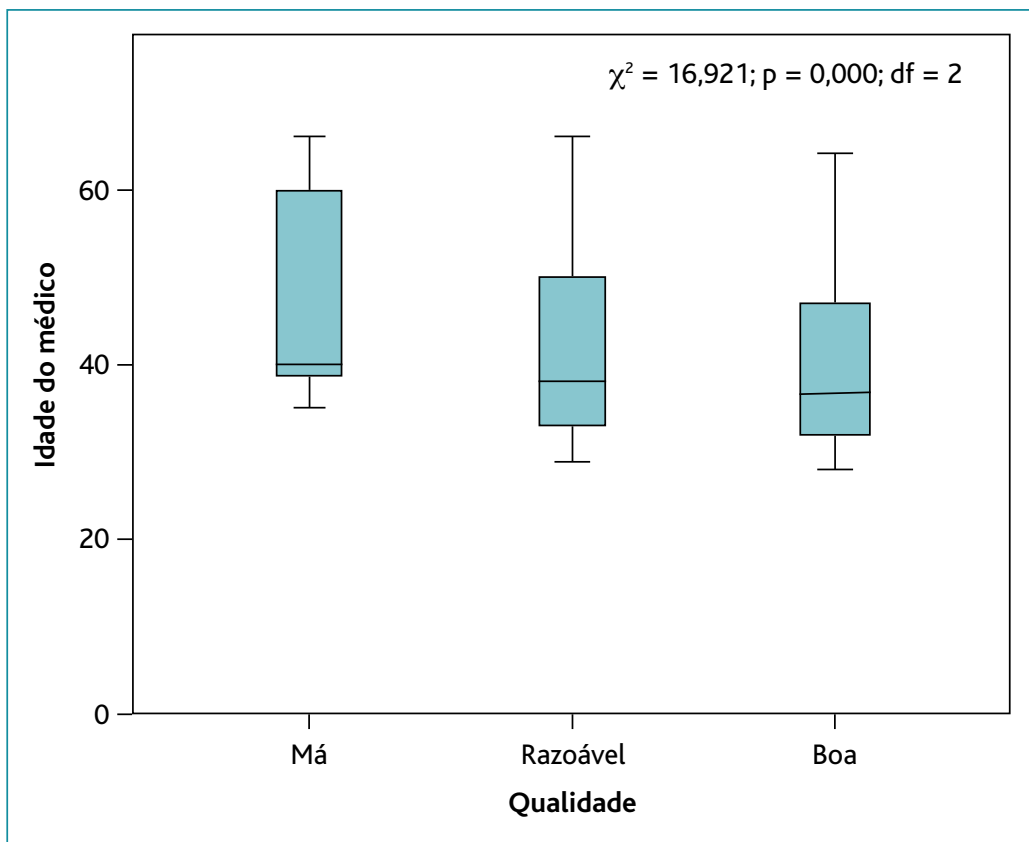

Figura 3. Associação entre a idade do médico referenciador e a qualidade da referenciação $\left[\chi^{2}=16,921 ; p=0,000 ; d f=2\right]$.

\section{Tempo de execução de triagem}

O Quadro IV representa o tempo de execução de triagem, de agendamento e de efetivação da consulta (mínimo, máximo, mediano) em dias, por cada especialidade da amostra de referenciações.

Da totalidade das referenciações, $75 \%$ obtiveram uma execução da triagem em 10 dias; ao fim de 30 dias, $90,6 \%$ tinham o processo de triagem concluído. Apesar disto, verificaram-se tempos máximos de triagem su-
O tempo mediano de agendamento de consulta hospitalar, considerando a globalidade dos pedidos de consulta da amostra, foi de 93 dias (AIQ=125; IC95\% [82-106]), sendo o tempo mediano para a efetivação da consulta hospitalar de 83 dias [AIQ=99 dias; IC95\% [73-97]). Globalmente verificou-se que o tempo mediano de efetivação de consulta foi inferior ao de agendamento, significando que durante o processo de referenciação houve uma antecipação da data da marcação das consultas. Este aspeto verificou-se, individualmente, nas especialidades de cardiologia, cirurgia geral/ambulatório, cirurgia plástica e reconstrutiva, dermatologia/rastreio teledermatológico, endocrinologia, ginecologia, neurologia, oftalmologia, ortopedia e pediatria. O inverso verificou-se nas especialidades de cirurgia geral, imunoalergologia, nefrologia, pneumologia, psiquiatria/consulta geral, especialidades em que o tempo de efetivação foi superior ao tempo de agendamento, pelo que no processo de marcação de consulta ocorreu o adiamento da data inicialmente prevista para a realização da consulta.

Ressalva-se ainda que as especialidades endocrinologia, dermatologia/rastreio teledermatológico, psiquiatria/consulta geral e psiquiatria da infância e da adolescência se encontram em regime de consultoria, significando que os tempos de agendamento e efetiva- 
ção da consulta são referentes à consultoria, isto é, consultas efetuadas sem a presença do utente.

No caso da dermatologia/rastreio teledermatológico verificou-se um tempo mediano de efetivação da consultoria (sem presença do doente) muito inferior ao tempo de agendamento, mas ressalva-se que das 26 consultorias agendadas, apenas 10 foram efetivadas.

\section{Motivo de não efetivação da consulta}

Das consultas que inicialmente foram agendadas, $16,8 \%(n=109)$ não foram efetivadas. Os principais motivos de não efetivação das consultas encontram-se representados na Figura 6. Entre os motivos contemplados em «Outros» $(n=14)$, oito devem-se ao facto de a consulta ainda não se ter realizado à data da colheita dos dados do estudo, dois por falecimento dos utentes em data anterior à da efetivação da consulta e quatro por motivos não apurados.

\section{DISCUSSÃO}

Taxa de referenciação

A taxa de referenciação ao hospital de referência foi de 7,7\%, superior à obtida no ano de 1994 no Estudo Europeu Sobre Referenciação em Cuidados Primários, ${ }^{6}$ que comparou diversos países, constatando, em Portugal, uma taxa de referenciação de 5,56\%. É igualmente superior à de um estudo realizado numa extensão rural do CS de Serpa, ${ }^{7}$ que reportou uma taxa de referenciação de 5,87\%, bem como num estudo observacional, avaliando a referenciação de três centros de saúde aos cuidados hospitalares em Madrid (5,8\%). ${ }^{10}$ No entanto, foi inferior à obtida no estudo efetuado no CS da Senhora da Hora em 2003 (10,1\%). ${ }^{9}$

Vários estudos têm demonstrado discrepância nas taxas de referenciação. ${ }^{5,10}$ Esta discrepância pode ser explicada pela diferente epidemiologia das patologias da região de abrangência de cada sistema de saúde, diversidade de oferta dos serviços hospitalares, características sociodemográficas dos utentes e características inerentes ao médico (formação, personalidade). ${ }^{10}$
Os autores do presente estudo consideram que a diminuição da taxa de referenciação face ao estudo de 2003 poderá traduzir maior capacidade de resolução de problemas por parte dos CSP, em Matosinhos, mas também eventuais dificuldades na acessibilidade aos cuidados hospitalares em determinadas especialidades, que condicionam, à partida, uma redução dos pedidos de consulta por conhecimento generalizado entre os MF de falta de resposta satisfatória, efetiva e atempada. De salientar que a determinação da taxa de referenciação, em 2003, se refere apenas a um CS e que foi determinada por processo manual, associando-se a eventuais problemas de informação díspar, consoante a fonte, o que poderá ter influenciado um resultado mais elevado, conforme discutido nesse estudo. ${ }^{9}$

Em maio de 2016, o Despacho n. ${ }^{\circ}$ 5.911-B/2016 aprovou o sistema de livre acesso e circulação de utentes no Serviço Nacional de Saúde (SNS), permitindo que a referenciação de um utente possa ser feita para qualquer uma das unidades hospitalares do SNS. Verificaram-se 4.746 referenciações do ACeS de Matosinhos a outras unidades hospitalares que não o hospital de referência, o que perfaz $13,5 \%$ do total de referenciações desse ano. Cerca de $65 \%$ das referenciações a outros centros hos- 
QUADRO III. Pedidos de consulta recusados por especialidade

\begin{tabular}{l|c|c} 
Especialidade & $\begin{array}{c}n \\
\text { pedidos }\end{array}$ & $\begin{array}{c}\text { \% de pedidos de consulta } \\
\text { recusados por especialidade }\end{array}$ \\
\hline Medicina física e reabilitação & 2 & 50,0 \\
\hline Cardiologia & 15 & 33,3 \\
\hline Hematologia clínica & 4 & 25,0 \\
\hline Imuno-hemoterapia & 5 & 20,0 \\
\hline Urologia & 25 & 20,0 \\
\hline Neurologia & 11 & 18,2 \\
\hline Gastrenterologia & 6 & 16,7 \\
\hline Anestesiologia & 7 & 14,3 \\
\hline Dermatologia/rastreio teledermatológico & 30 & 13,3 \\
\hline Ginecologia & 41 & 7,3 \\
\hline Pneumologia & 14 & 7,1 \\
\hline Oftalmologia & 127 & 6,3 \\
\hline Cirurgia plástica e reconstrutiva & 16 & 6,3 \\
\hline Medicina interna & 17 & 5,9 \\
\hline Obstetrícia & 19 & 5,3 \\
\hline Cirurgia geral & 68 & 4,4 \\
\hline Ortopedia & 93 & 3,2 \\
\hline Otorrinolaringologia & 58 & 1,7 \\
\hline
\end{tabular}

Fonte: ALERT - CTH ${ }^{\circledR}$.

pitalares foram feitas para especialidades não disponíveis no $\mathrm{HPH}$, na sua maioria angiologia e cirurgia vascular $(n=1.120)$, estomatologia $(n=822)$, neurocirurgia ( $n=378)$, cirurgia pediátrica $(n=329)$ e cirurgia geral/obesidade $(n=191)$.

Este facto também poderá ter contribuído para uma diminuição da taxa de referenciação ao hospital de referência, embora o volume de referenciações para outras instituições, em especialidades disponíveis no $\mathrm{HPH}$, seja diminuto - apenas $5 \%$ do total de referenciações do ano 2016.

\section{Motivos de referenciação}

Os principais motivos de referenciação deste estudo foram o diagnóstico e tratamento, resultados consistentes com outros estudos. ${ }^{10-11}$ A pressão exercida pelos utentes surge como um importante motivo de referenciação atualmente, consequência da maior literacia em saúde e da informação transmitida pela comunicação social. ${ }^{5,10}$ Verifica-se que os médicos sentem algum grau de pressão por parte dos utentes para serem referenciados em 18,3\% dos casos. ${ }^{10}$ Também a prática de uma medicina mais «defensiva" por parte dos médicos, temendo potenciais consequências legais associadas a uma maior informação em saúde por parte dos utentes, constitui um motivo de referenciação, mas que no presente estudo não foi possível mensurar., ${ }^{5,12}$ O estudo de Donohoe e colaboradores mostra que os MF referenciam por motivos médicos e não médicos e que a opinião deles é que se tivessem mais experiência em determinados procedimentos ou melhor rede de comunicação com as especialidades hospitalares poderiam ser evitadas referenciações, com redução de custos. ${ }^{5}$ Nesse estudo houve discordância entre MF e médicos hospitalares em relação às referenciações «evitáveis» em $34 \%$ das mesmas. A necessidade de consenso e uniformização dos motivos de referenciação pode, no futuro, vir a suscitar melhores práticas a nível do processo de articulação de cuidados.

O presente estudo demonstrou que a maioria dos utentes referenciados eram mulheres, em consonância com outros estudos, ${ }^{10} \mathrm{o}$ que pode relacionar-se com o facto de as mulheres procurarem mais os cuidados de saúde.

\section{Principais especialidades referenciadas}

No presente estudo as principais especialidades solicitadas foram cirúrgicas (oftalmologia, ortopedia e cirurgia geral), as quais abordam patologias que necessitam de técnicas específicas para diagnóstico e tratamento, não disponíveis nos CSP, justificando a necessidade de maior referenciação. Este resultado foi sobreponível a outros estudos, ${ }^{6-8,10-11,13}$ incluindo o realizado no CS da Senhora da Hora em 2003, ${ }^{9}$ variando 


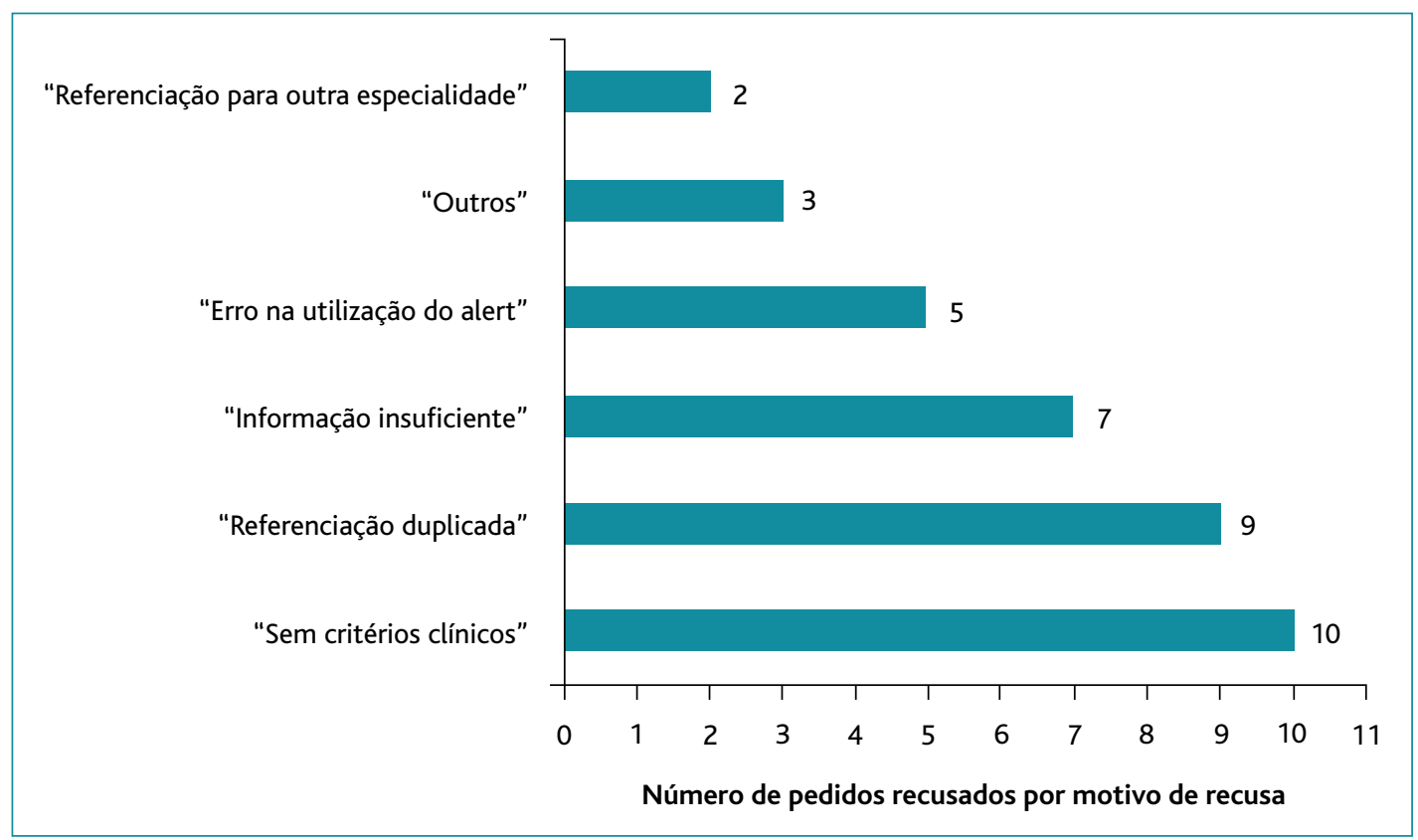

Figura 5. Motivos de recusa de agendamento de consulta por parte dos diferentes serviços hospitalares do Hospital Pedro Hispano.

apenas o tipo de especialidade cirúrgica solicitada, o que poderá sugerir ter-se obtido uma melhoria na resposta e oferta de cuidados por determinadas especialidades, aumentando a sua referenciação pelos MF particularmente visível na especialidade de oftalmologia, outrora com poucos pedidos de consulta pelos MF, uma vez que apresentava baixa capacidade de resposta, com tempos de espera muito prolongados.

\section{Informatização do sistema de referenciação e a qualidade das cartas}

A informatização do sistema de referenciação aos cuidados hospitalares, via ALERT-CTH®, bem como a generalização da efetivação dos registos clínicos no SClínico ${ }^{\circledR}$ Hospitalar por parte das especialidades hospitalares, melhoraram determinados parâmetros da qualidade das referenciações, possibilitando a monitorização da resposta hospitalar e a obtenção da informação clínica de retorno através dos registos informáticos no SClínico® Hospitalar (atualmente quase sempre presentes, mas praticamente inexistentes em 2003, quando apenas foi possível encontrar informação clínica de retorno escrita em $26,3 \%$ dos processos clínicos, em papel, consultados). ${ }^{9}$
De referir a presença de respostas clínicas hospitalares no ALERT-CTH® em alguns casos. É de salientar que este sistema, não estando preparado para a devolução de respostas, não dando alerta destas ao MF, pode incorrer em risco clínico aquando da sua utilização para esta função, uma vez que o MF pode não ficar ciente desta informação em tempo útil para fornecer uma resposta atempada ao utente.

Comparativamente ao estudo efetuado no mesmo contexto e publicado em $2006,{ }^{9}$ a melhoria dos sistemas de informação tornou o processo de referenciação mais transparente, melhorou a identificação e a legibilidade da informação das cartas de referenciação e permitiu maior discriminação dos tempos de espera para efetivação da consulta. Este facto reflete-se no presente estudo, que demonstrou uma melhoria da qualidade das cartas de referenciação $(31,1 \%$ com boa qualidade e $66,3 \%$ com razoável qualidade) comparativamente ao estudo de 2003, que mostrou apenas $9,4 \%$ de cartas com boa qualidade e $65,3 \%$ com razoável qualidade. ${ }^{9}$ De salientar que os critérios de qualidade utilizados foram semelhantes entre os dois estudos, com as adaptações necessárias à evolução do processo de referenciação, utilizando um sistema informático. 


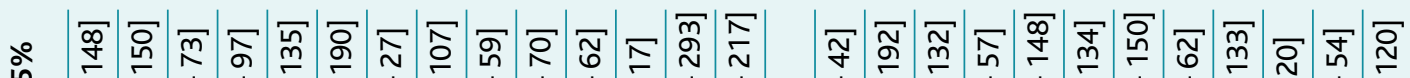

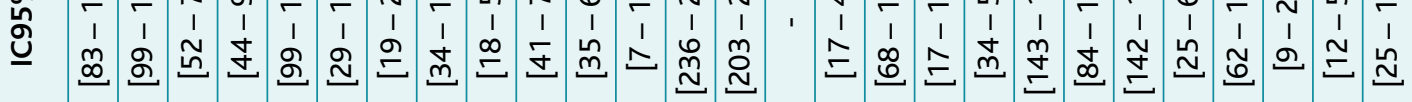

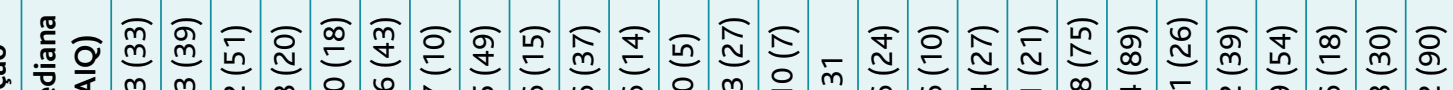

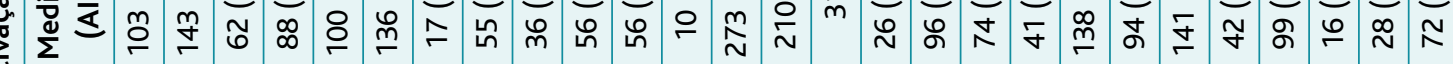
总

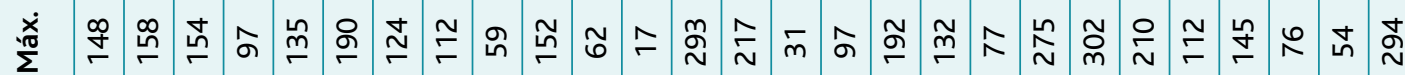

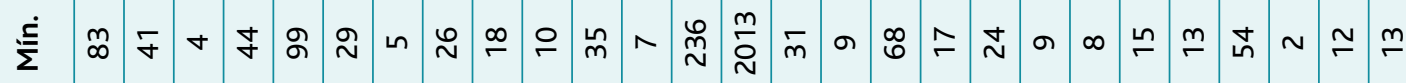

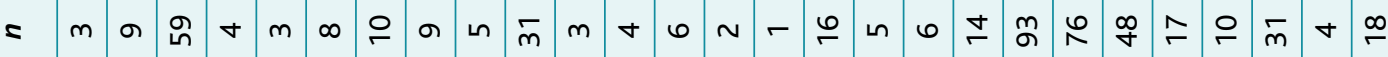

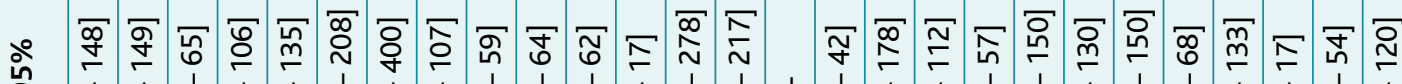

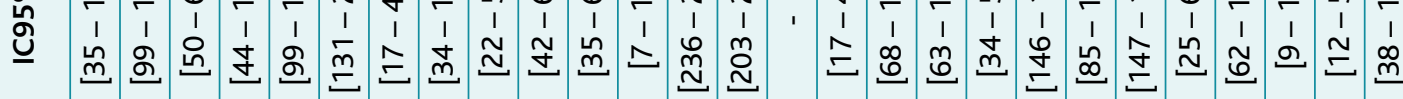

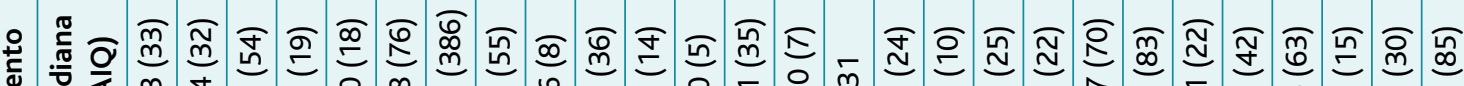

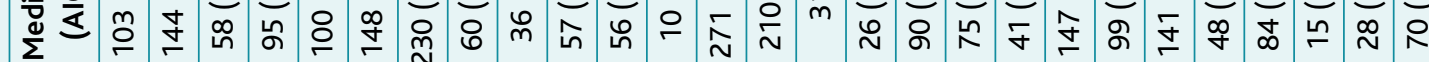

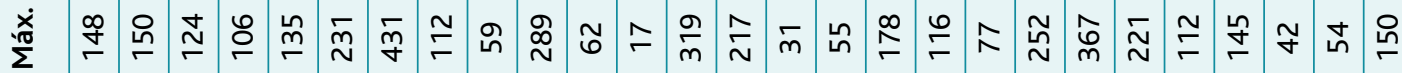

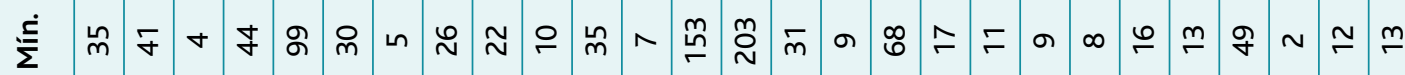

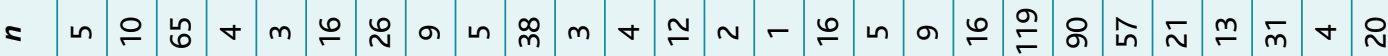

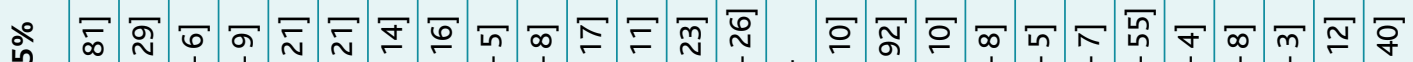

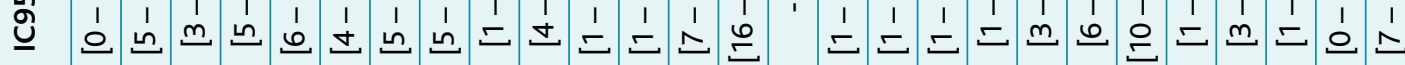

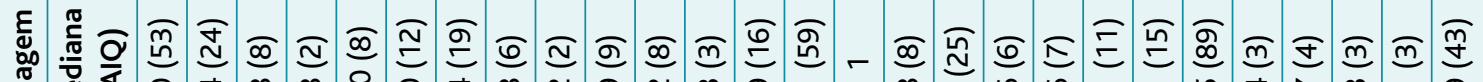
¿

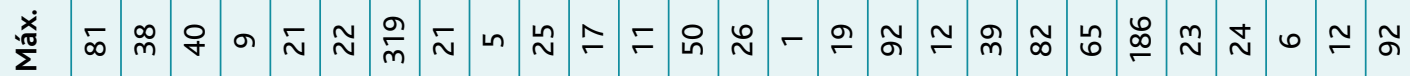

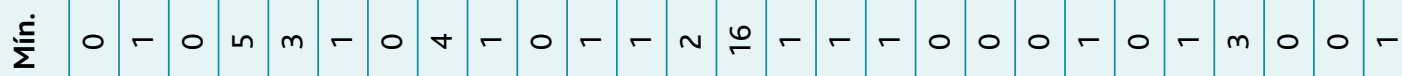

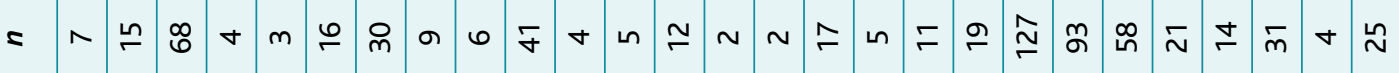




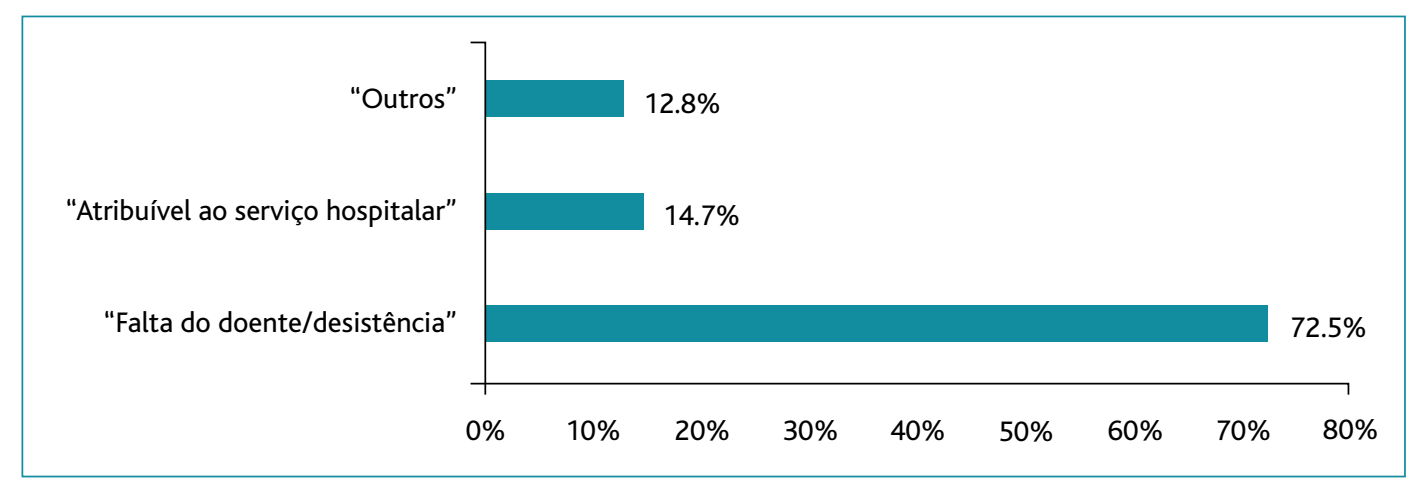

Figura 6. Principais motivos de não efetivação das consultas.

Estudando a qualidade da prática clínica dos MF em Inglaterra, Foot e colaboradores verificaram que a base de uma referenciação com qualidade aos cuidados hospitalares implica: necessidade de referenciação (se e quando?); escolha de momento adequado (precoce ou atraso evitável?); destino (especialidade adequada); e processo de referenciação de elevada qualidade (informação necessária em formato claro; escolha informada e partilhada com utente do momento e local de referenciação; MF, utente e médico hospitalar concordam com propósito da referenciação). ${ }^{4} \mathrm{O}$ College of Family Physicians of Canada (CFPC) e o Royal College of Physicians and Surgeons of Canada (Royal College) consideram que a informação mais importante que deve constar na carta de referenciação deve ser: informação sobre o problema que motiva a referenciação, questões/dúvidas clínicas; detalhes sobre o utente que é improvável que ele refira na consulta ou não saiba e que sejam importantes do ponto de vista clínico; problemas de saúde concomitantes; medicação crónica; exames complementares de diagnóstico ou tratamentos já efetuados para o problema atual. ${ }^{15} \mathrm{~A}$ literatura refere que existe elevada discrepância entre os MF e os médicos hospitalares no que respeita à definição de qualidade das cartas de referenciação. ${ }^{16}$ Atendendo a estes factos parece existir a necessidade de consensos/critérios para uniformizar critérios de qualidade das cartas de referenciação, com eventual criação de protocolos de referenciação. A melhoria da formação médica pré-graduada e contínua bem como das políticas de referenciação locais, com protocolos bem definidos, sistemas informáticos amigáveis e facilitadores poderão levar, na opinião dos autores, a uma melhoria das cartas de referenciação. Neste estudo demonstrou-se uma associação inversa entre a idade dos médicos e a qualidade das cartas, conforme expresso na Figura 3, o que pode traduzir a maior acessibilidade dos médicos mais jovens aos fatores atrás enunciados.

\section{Tempos de espera até à consulta nos cuidados hospitalares}

Também se verificou uma melhoria dos tempos de espera até à consulta, com um tempo mediano de 84 dias, relativamente ao tempo de espera verificado em 2003 (entre um a seis meses). Estes tempos variam consoante a especialidade, sendo que se observam casos em que os tempos medianos são superiores a 120 dias - tempo máximo de espera até à efetivação da primeira consulta hospitalar, estabelecido pela legislação em vigor desde janeiro de 2018. ${ }^{14}$ Assim, torna-se necessária uma reorganização célere dos serviços.

Discussão dos pontos fortes e pontos fracos do estudo

O presente estudo destaca-se pela sua robustez e validade interna, sendo importante verificar em que medida é que os resultados podem ser semelhantes em outros contextos, com diferentes respostas hospitalares e diferente contexto de CSP. Foi o primeiro estudo que avaliou todo o trajeto desde a referenciação à efetivação da consulta hospitalar com monitorização do tempo de triagem, tempo de agendamento e tempo de efetivação. Decidiu-se analisar possíveis associações entre a idade dos médicos referenciadores e a qualidade das cartas, com os tempos até à efetivação da consulta, no pressuposto de que cartas com maior qualidade e melhor descrição do quadro clínico e motivo de refe- 
renciação poderiam justificar maior celeridade na marcação. Trata-se de um preconceito decorrente da prática clínica que os autores pretenderam verificar, tendo-se obtido uma associação entre as variáveis. Poder-se-ia supor, igualmente, que a idade (como surrogate de experiência clínica/atualização/formação) tivesse interferência na qualidade das cartas e, portanto, na celeridade da marcação. Não foi encontrado nenhum estudo anterior que avaliasse e demonstrasse associação da qualidade das cartas de referenciação com a idade dos médicos e o tempo de efetivação da consulta, pelo que estes resultados necessitam de outras investigações que os possam corroborar.

Um dos pontos fortes deste estudo foi a estratégia de amostragem e a análise de cada referenciação com vista a ultrapassar as limitações iniciais com que os investigadores se depararam (viés de registo), inerentes a erros detetados nos processos de agendamento e utilização do ALERT-CTH®, nomeadamente alterações de datas de agendamentos sucessivas, sem fundamentação clara e motivos de recusa não objetivos. Uma limitação do estudo é a possibilidade de viés de observador. Contudo, de forma a minimizá-lo, foi realizada uma análise conjunta de $5 \%$ das referenciações, reuniões de consenso intercalares para uniformização de critérios, bem como análise e revisão conjunta, no final, dos casos que suscitaram dúvidas.

\section{CONCLUSÕES}

Neste estudo foi possível determinar a taxa de referenciação ao principal hospital de referência por parte de um ACeS, ambos inseridos numa Unidade Local de Saúde, através de um sistema de referenciação informatizado e analisar a qualidade das cartas de referenciação, fazendo uma comparação com estudo efetuado no mesmo contexto, treze anos antes, previamente à informatização generalizada do processo de referenciação. Determinaram-se também quais os tempos de resposta hospitalar e analisaram-se variáveis como a menor idade dos médicos referenciadores e a maior qualidade das cartas de referenciação, que demonstraram ter uma associação estatisticamente significativa com os tempos de resposta hospitalar.

A finalidade deste estudo é melhorar a articulação entre os diferentes níveis de cuidados no processo de referenciação. Os autores sugerem a integração do pro- cesso de referenciação no SClínico®, tornando-o um sistema único capaz de minimizar a perda de informação, de melhorar a qualidade da informação das cartas de referenciação e reduzindo o tempo despendido pelos MF no processo de referenciação usando dois sistemas; seria facilitador para o MF e objetivaria a prestação de cuidados mais eficazes e de maior qualidade e transparência.

O presente estudo abre pistas para uma análise da rentabilidade do regime de consultoria, salientando a necessidade de estudos futuros neste âmbito, concretamente na avaliação da taxa de execução de consulta, «tempo poupado», satisfação de utentes e profissionais com este processo, que deixa antever diversas insuficiências a todos os níveis.

\section{REFERÊNCIAS BIBLIOGRÁFICAS}

1. WONCA Europa. A definição europeia de medicina geral e familiar (Clínica geral/Medicina familiar).WONCA Europa; 2002. Available from: http://www.woncaeurope.org/sites/default/files/documents/European\%20Definition\%20in\%20Portuguese.pdf

2. Freeman TR, revised editor. McWhinney's textbook of family medicine. New York: Oxford University Press; 2016. ISBN 9780199370689

3. General Practitioners Committee. Good medical practice for general practitioners [Internet]. London: Royal College of General Practitioners; 2008. ISBN 9780850843224. Available from: https://www.lmc.org.uk/ visageimages/guidance/2008/rcgp_good_medical_practice_for_gps_jul y_2008.pdf

4. Foot C, Naylor C, Imison C. The quality of GP diagnosis and referral [Internet]. London: London: The King's Fund; 2010. Available from: https://www.kingsfund.org.uk/sites/default/files/Diagnosis\%20and\%2 Oreferral.pdf

5. Donohoe MT, Kravitz RL, Wheeler DB, Chandra R, Chen A, Humphries $N$. Reasons for outpatient referrals from generalists to specialists. J Gen Intern Med. 1999;14(5):281-6.

6. Fleming DM. The European study of referrals from primary to secondary care [dissertation] [Internet]. Amsterdam: Thesis Publishers; 1993. Available from: https://cris.maastrichtuniversity.nl/ws/files/ 1354488/guid-65634c73-8849-4755-a05b-7cf1d976af6d-ASSET1.0

7. Janeiro M. Acesso aos cuidados de saúde secundários numa extensão do Centro de Saúde de Serpa: 10 anos depois [Access to secondary care from Serpa Health Centre]. Rev Port Clin Geral. 2001;17(3):193-207. Portuguese

8. Barreiro S. Referenciação e comunicação entre cuidados primários e secundários [Referral and communication between primary and secondary care]. Rev Port Clin Geral. 2005;21(6):545-53. Portuguese

9. Ponte CM, Moura BG, Cerejo AC, Braga R, Marques I, Teixeira A, et al. Referenciação aos cuidados de saúde secundários [Referral to secondary care]. Rev Port Clin Geral. 2006;22(5):555-68. Portuguese

10. de Prado Prieto L, Garcia Olmos L, Rodríguez Salvanés F, Otero Puime A. Evaluación de la demanda derivada en atención primaria [Evalua- 
tion of referrals in primary care]. Aten Primaria. 2005;35(3):146-51. Spanish

11. Forrest $C B$, Nutting PA, Starfield B, von Schrader S. Family physicians referral decisions: results from the ASPN referral study. J Fam Pract. 2002;51(3):215-22.

12. Xu T, Rohrer J, Borders T. The impact of managed care and practice size on primary care physicians' perceived ability to refer. J Health Serv Res Policy. 2002;7(3):143-50.

13. García Olmos L. Análisis de la demanda derivada en las consultas de medicina general en España [dissertation] [Internet]. Madrid: Universidad Autónoma de Madrid; 1993. Available from: https://repositorio.uam .es/handle/10486/141

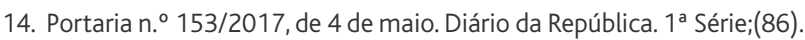

15. College of Family Physicians of Canada, Royal College of Physicians and Surgeons of Canada. Guide to enhancing referrals and consultations between physicians [homepage]. Mississauga: RFPC; 2009. Available from: https://www.cfpc.ca/ProjectAssets/Templates/Resource.aspx?id $=3448$

16. Westerman RF, Hull FM, Bezemer PD, Gort G. A study of communication between general practitioners and specialists. $\mathrm{Br} J$ Gen Pract. 1990;40(340):445-9.

\section{CONFLITO DE INTERESSES}

Os autores declaram não ter quaisquer conflitos de interesse.

\section{ENDEREÇO PARA CORRESPONDÊNCIA}

Hugo Rocha

E-mail: hugofp.rocha@gmail.com

https://orcid.org/0000-0003-3940-606X

Recebido em 02-02-2018

Aceite para publicação em 08-05-2019

\section{ABSTRACT}

\section{REFERRAL TO HOSPITAL CARE USING A COMPUTER SYSTEM}

Objectives: In 2003, a study carried out at Senhora da Hora Health Center, prior to computerization of the referral system, found a hospital referral rate of $10.1 \%$, with referral letters mostly of medium quality and a mean waiting time for consultation ranging from one to six months. This study aims to determine the referral rate to Pedro Hispano Hospital (HPH) by all family physicians from the ACeS de Matosinhos in 2016, to evaluate the quality of referral letters, to analyze response times of secondary health care and to assess the relationship between the age of family physicians, the time of consultation and the quality of referrals.

Methods: Cross-sectional, analytical study, conducted in Matosinhos' Local Health Unit. We analyzed the referral letters for patients referred to HPH during the year 2016. Simple random sample, representative of the study population $(n=649)$. The analysis used descriptive and analytical statistics (Kruskall-Wallis).

Results: The referral rate was $7.7 \%$. The median age of the referred population was 53 years, $53.7 \%$ of the patients were female. The specialties with the greatest number of referrals were ophthalmology $(18.0 \%)$, orthopedics $(14.4 \%)$ and general surgery (9.9\%). About $54 \%$ of the referrals were for diagnosis. The referral letters were judged to be of reasonable quality $(66.3 \%)$ and of very good quality (31.1\%). $6.6 \%$ of the referrals were refused, because of the lack of clinical criteria for referral. Of all the letters, $90.6 \%$ were screened within 30 days. The quality of the referral letter was associated with more rapid consultation $(p=0.003)$. Discussion: Thirteen years after an initial study of referral in this population, the following changes were noted: transparency in the referral process and improvements in the identification and readability of the information of the reference letters. There was an increase in the number of referrals with reasonable and very good quality. There were changes in the most referred specialties, suggesting that, with an increase in the supply of care, there is a greater demand. The computerization of the referral process allowed greater discrimination of waiting times for hospital appointments. A strength of this study was the ability to track of the booking of hospital appointments, with monitoring of the times of screening, scheduling, and the date of hospital appointments, overcoming limitations due to errors detected in the use of computerized referral software. This study has internal validity and it is important to evaluate to what extent the results may be similar in other contexts.

Keywords: Referral and consultation; Primary health care; Secondary care; Hospitals. 